\title{
Protée
}

\section{Louis-Pierre Bougie}

Le corps est la demeure du temps

\section{Michaël La Chance}

Volume 34, numéro 2-3, automne-hiver 2006

Actualités du récit. Pratiques, théories, modèles

URI : https://id.erudit.org/iderudit/014269ar

DOI : https://doi.org/10.7202/014269ar

Aller au sommaire du numéro

\section{Éditeur(s)}

Département des arts et lettres - Université du Québec à Chicoutimi

ISSN

0300-3523 (imprimé)

1708-2307 (numérique)

Découvrir la revue

Citer cet article

Chance, M. L. (2006). Louis-Pierre Bougie : le corps est la demeure du temps Protée, 34(2-3), 103-112. https://doi.org/10.7202/014269ar d'utilisation que vous pouvez consulter en ligne.

https://apropos.erudit.org/fr/usagers/politique-dutilisation/ 


\section{LOUIS-PIERRE BOUGIE}

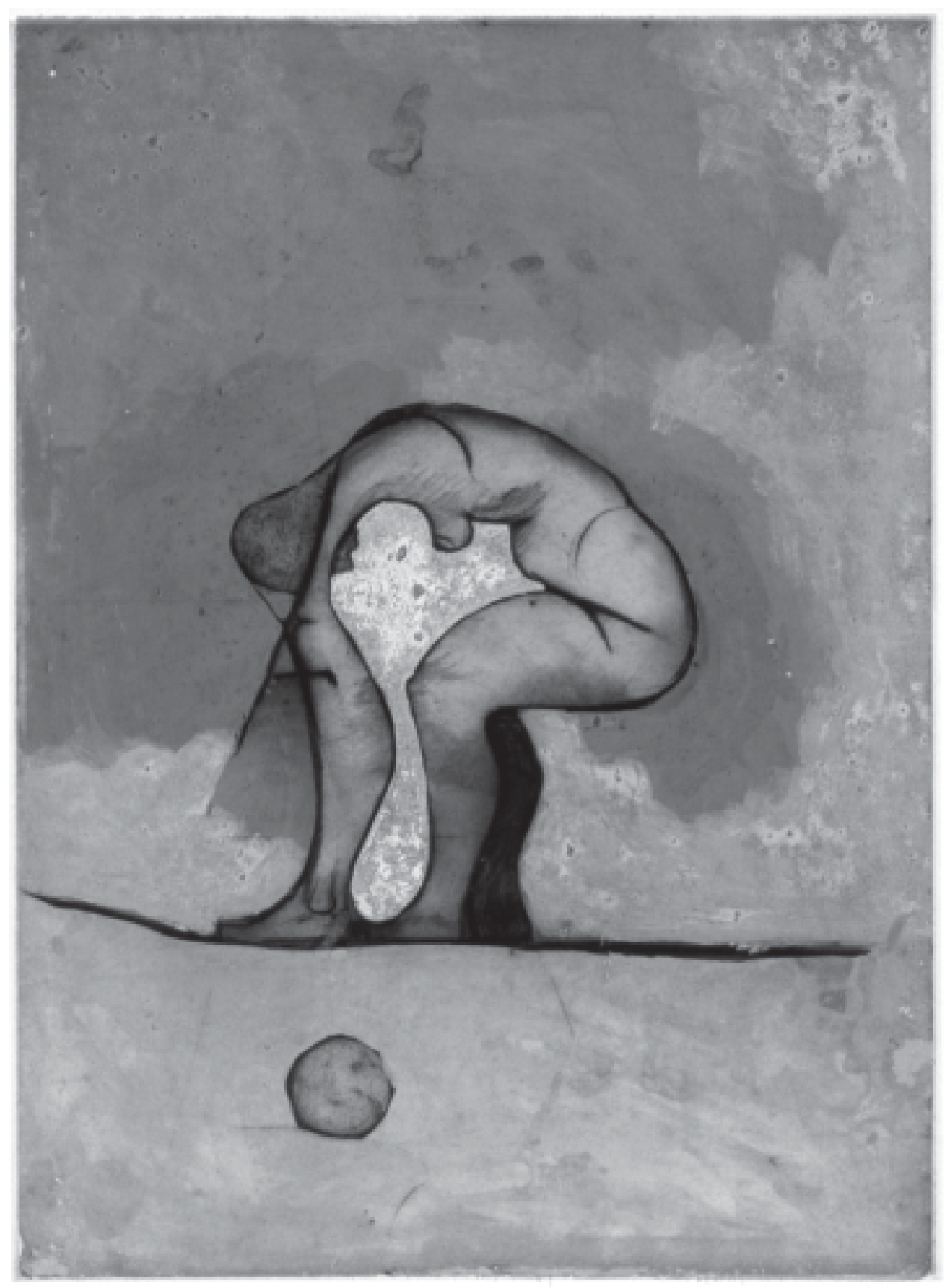

Suite Finlandaise, 2004, 65 x $50 \mathrm{~cm}$. 


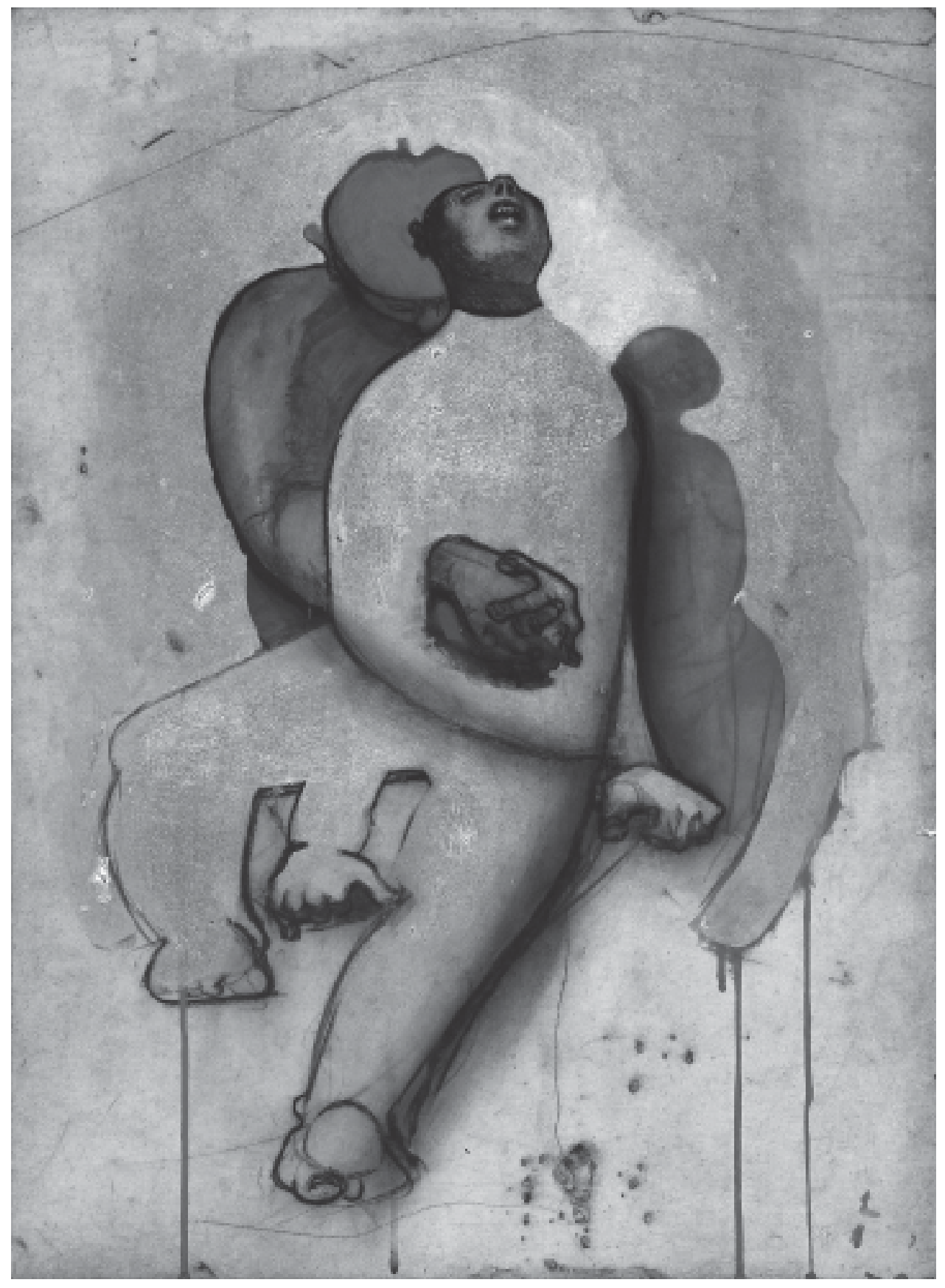

Travail de serre, 2005, 65 x $50 \mathrm{~cm}$. 


\section{LE CORPS EST LA DEMEURE DU TEMPS}

Ce que nous appelons le «corps» nous dit quelque chose du corps, mais cela met en scène bien d'autres choses, comme la manière dont l'esprit se représente à lui-même. Le corps, lorsqu'il paraît inachevé, larvaire, handicapé, est sans doute l'expression d'un état de l'esprit, sinon d'une condition de l'être: son ouverture sur le monde ou, au contraire, son repli sur lui-même. Ainsi, notre corps serait tantôt un puits d'émotion, dans lequel nous pouvons puiser des hurlements, tantôt une surface sur laquelle nous pouvons jouer avec des sentiments empruntés: le corps prend chaque fois la forme de ses affects. Chez Louis-Pierre Bougie, il y a un mouvement tournant du corps qui entraîne une torsion de l'espace: nous avons la révélation de la relation du corps à l'espace, le corps se saisit de l'espace dans lequel il se produit et fore dans celui-ci une indistinction du dedans et du dehors, du possible et de l'actuel. Nous avons appelé de telles figures des toponiries, car elles refusent de disjoindre l'expérience de l'espace et la manière dont les images surgissent, se dissipent, clignotent, flottent, se recomposent et se dissipent encore - sur le modèle de la coïncidence des contraires et de l'écartèlement de l'identique dans le rêve. Dessiner, c'est alors une façon de nous demander par quelle extrémité de nous-mêmes, perdus dans quelque coma matériel, nous sommes aussi une image dans un rêve lointain.

La production récente de Louis-Pierre Bougie travaille aussi la matière du temps dans les corps. En premier lieu, le temps nous semble un déroulement continu et nous lui prêtons une pureté géométrique. Mais, bientôt, le temps se révèle un fuseau d'univers dans lequel le début et la fin se touchent, dans lequel les répétitions - et aussi les premières occurrences - laissent des tracés. Où tous les événements sont déjà dans les plis, où la mémoire attend ses contours. Le temps n'est alors pas lisse et sans bord - comme on le dit de l'espace -, il est un espace qui se souvient, c'est un temps où chaque geste, à peine échappé d'un tourbillon laiteux, entre dans une ombre de cendres. C'est un temps strié, rugueux, fissuré - il est partout, dans la structure de nos pensées, dans la tension interne de notre vie et, aussi, dans la lecture que vous faites de ce texte.

Notre conception de la réalité s'est déplacée: elle sera faite non plus de particules matérielles, mais plutôt de perceptions partagées selon nos liens de communication et les probabilités sur lesquelles on s'accorde. Dans ce nouveau paradigme, la réalité tire sa consistance non pas d'une matière inerte, mais de la durée de ceux qui vivent dans cette réalité et de leurs projections organiques et 
symboliques. En fait, les formes, les sons, les couleurs - ce qui résiste et ce qui jaillit dans le monde dit «extérieur» - demeurent vides tant que le temps ne s'est pas inséré dans les objets. Lorsque le temps se laisse absorber dans les matières et concentrer dans les êtres, ces derniers comme les formes, les objets et les matières acquièrent une densité. Inversement, lorsque le temps s'échappe des corps, ils perdent toute consistance. Le ralentissement est grisaille, la couleur requiert une précipitation, il suffit de quelques feuilles vertes pour tinter le clair rappel d'une instantanéité. Il en va ainsi de l'âge glaciaire du sens, de la veille immobile des gardiens de la vie et du scintillement infinitésimal des minutes et des secondes accompagnant, jusqu'au dernier seuil, les grands véhicules qui descendent dans les profondeurs de l'Oubli.

Les dessins et peintures de Louis-Pierre Bougie s'attardent à observer la lente dissipation du temps hors des objets et des corps, ils présentent un miroir au souffle du temps. Ces œuvres tentent de décrire ce qui n'a pas eu lieu, une chose à laquelle la description même donne lieu - mais pas complètement, car la seule description des choses a pour effet de compresser le temps pour les générations futures. Un signe peut contenir plusieurs époques, un geste peut capturer une éternité. Alors le dessin est déjà une façon d'intervenir directement sur nos réalités, qui seront toujours tumulte de descriptions éphémères et, aussi, étau de souffrance.

Ici, en effet, le corps est la demeure du temps.

Michaël La Chance

Les photographies des œuvres de Louis-Pierre Bougie sont de Daniel Roussel. 


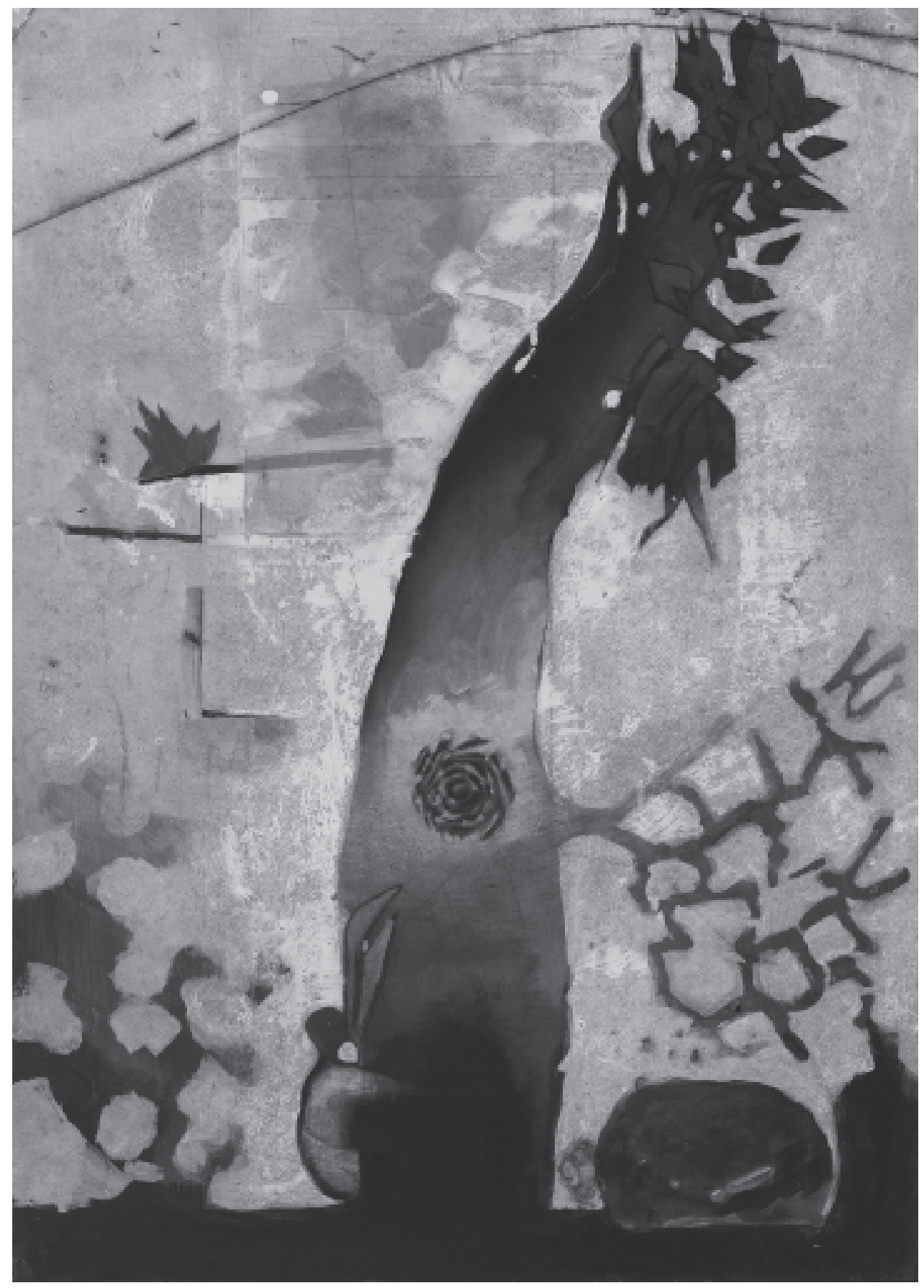

Le Baiser du temps, 2005, $65 \times 50 \mathrm{~cm}$. 


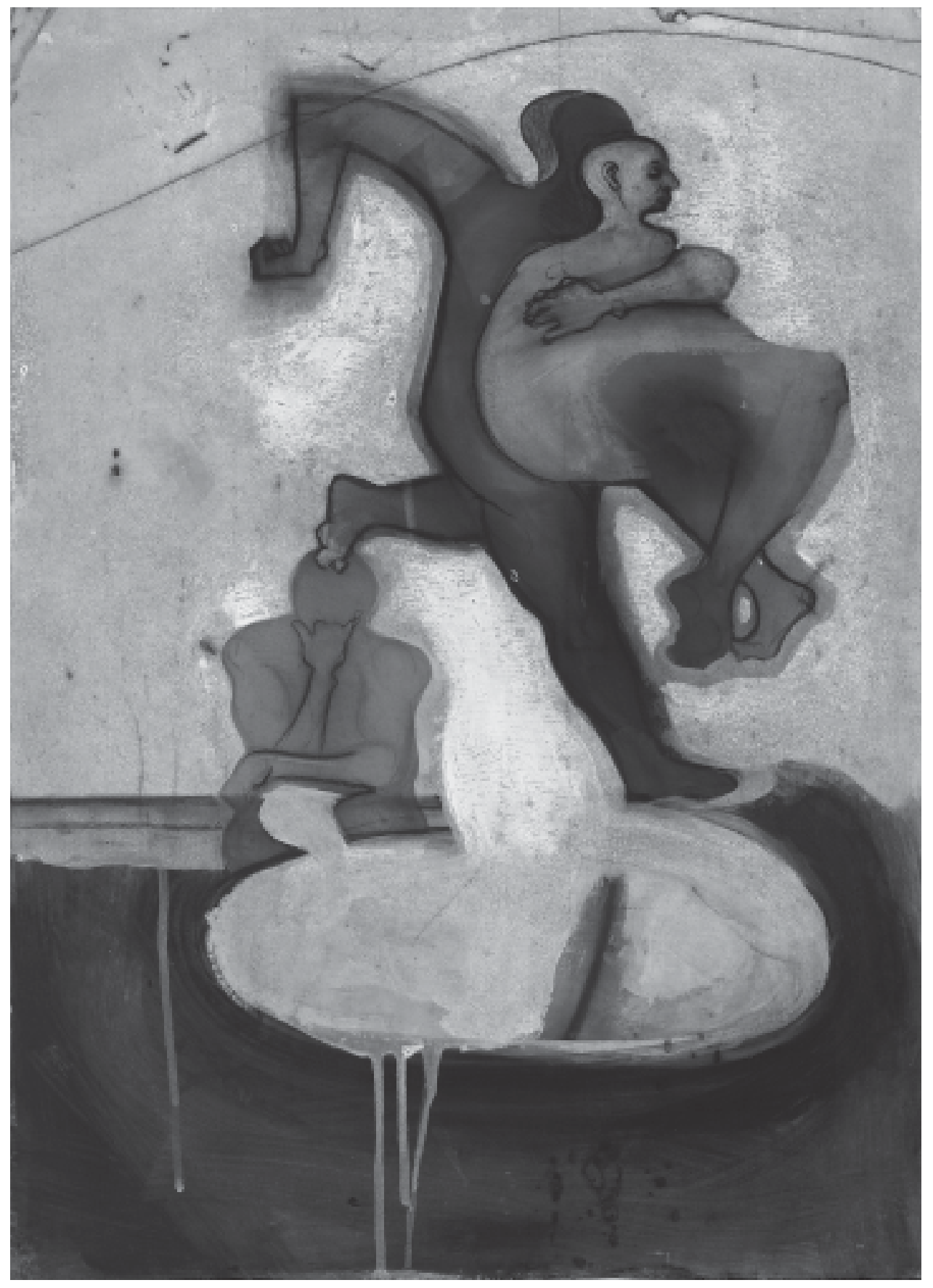

Le Perce-sommeil, 2005, 65 x $50 \mathrm{~cm}$. 


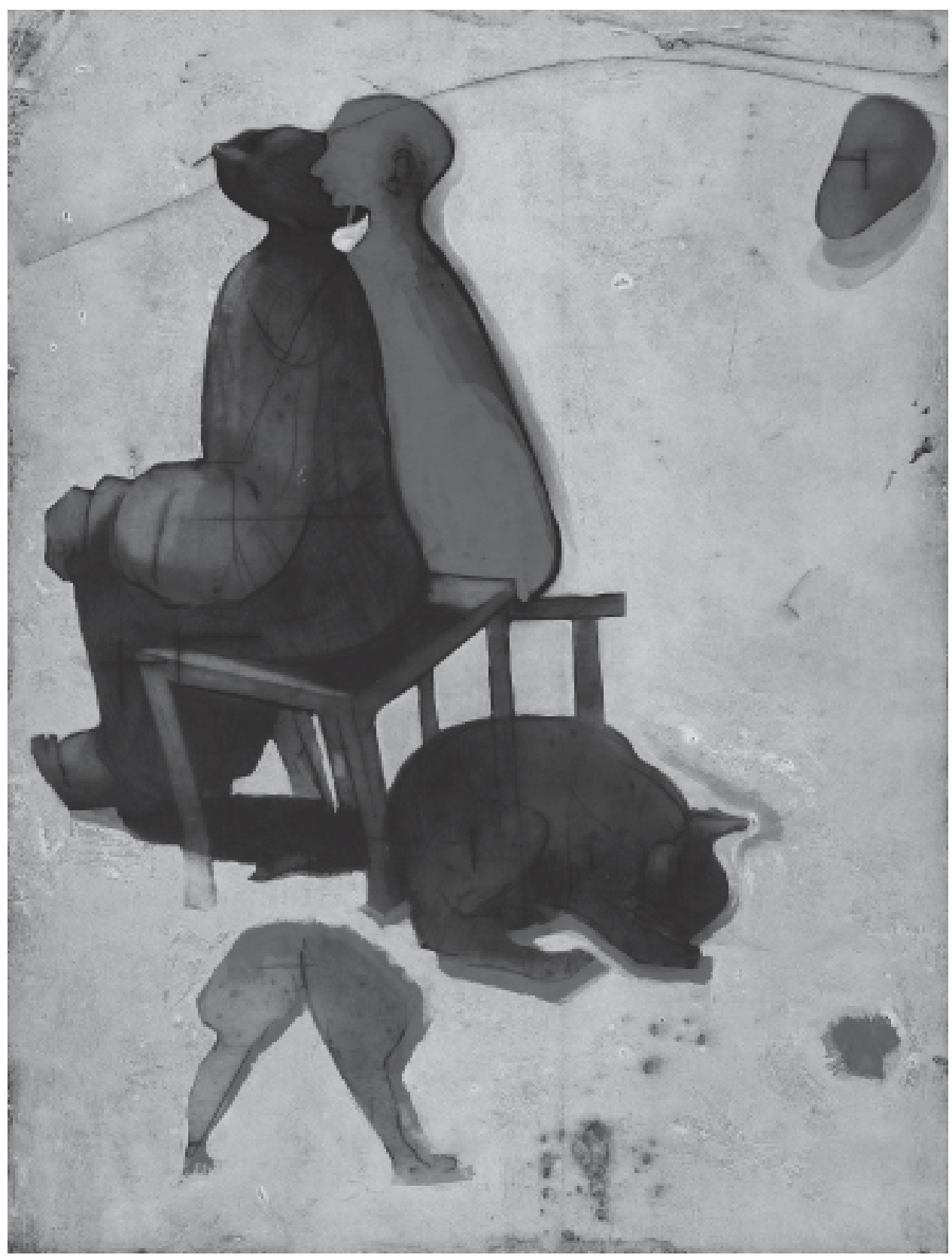

Germination, 2005, 65 x $50 \mathrm{~cm}$. 


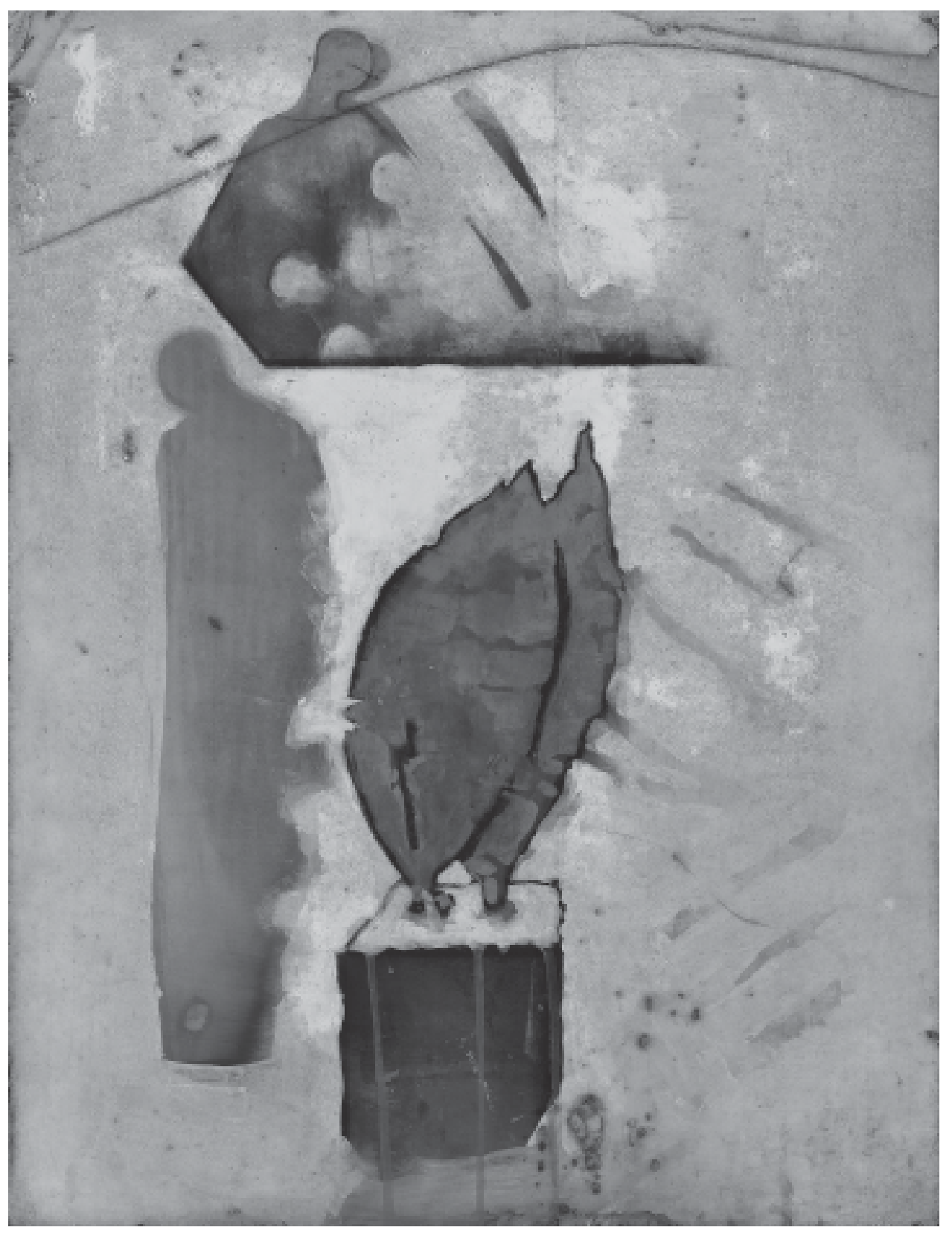

Possession, 2005, $65 \times 50 \mathrm{~cm}$. 


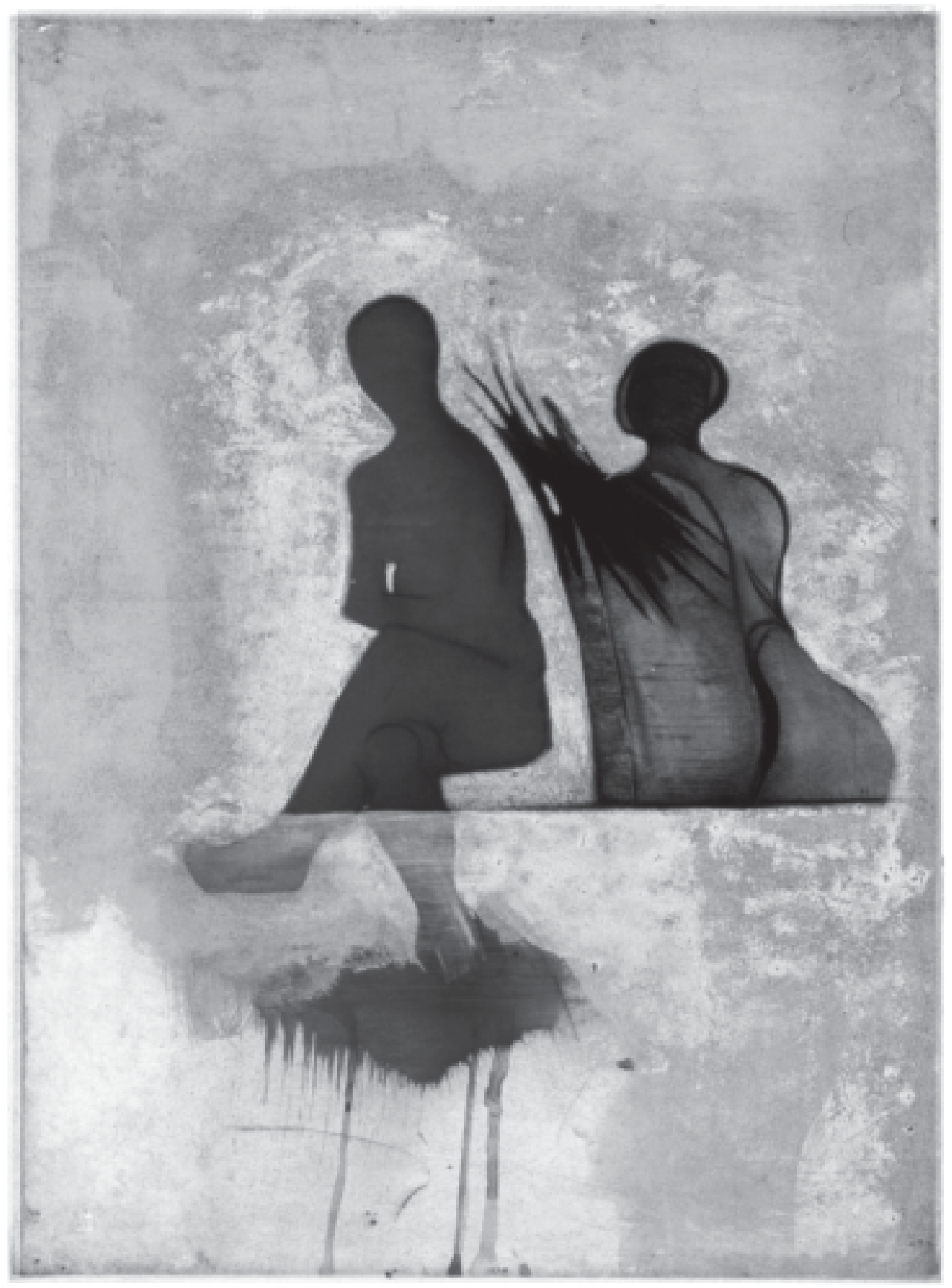

Suite Finlandaise, 2004, 65 x $50 \mathrm{~cm}$. 


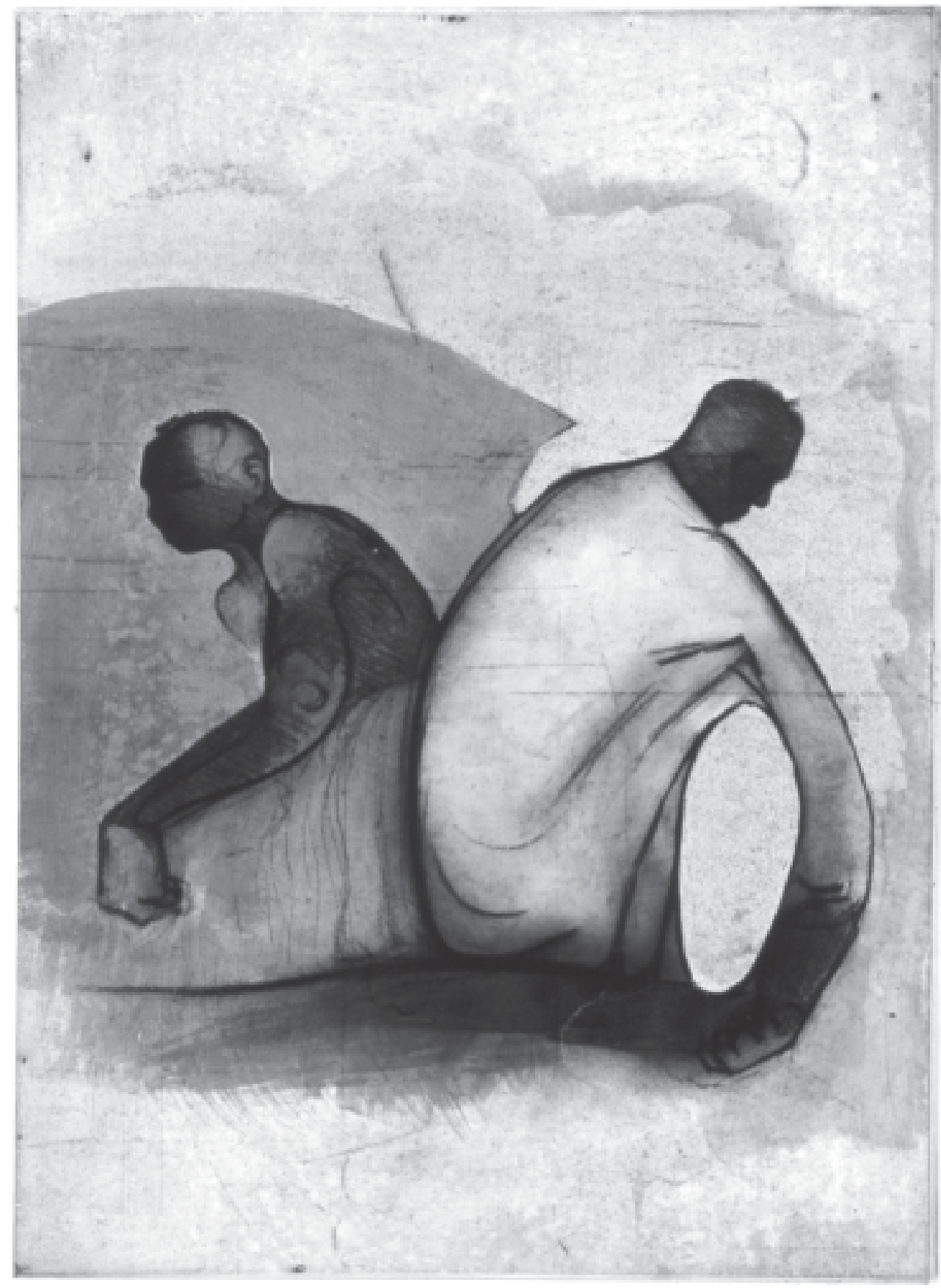

Suite Finlandaise I, 2004, 65 x $50 \mathrm{~cm}$. 